\title{
Web platform architecture for ambient assisted living
}

\author{
Ioana Stefan ${ }^{\mathrm{a}, \mathrm{b}, *}$, Constantin Lucian Aldea ${ }^{\mathrm{a}, \mathrm{b}}$ and Cosmin Septimiu Nechifor ${ }^{\mathrm{a}}$ \\ ${ }^{a}$ Siemens Corporate Technology, Bulevardul Eroilor No. 3A, 500007 Brasov, Romania \\ b Transilvania University of Brasov, Bulevardul Eroilor No. 29, 500036 Brasov, Romania
}

\begin{abstract}
Current demographic changes have made it necessary to implement innovative solutions in order to respond to elderly people's needs. This paper describes a concept designed to cater for such users' needs via modern technologies. Survey data and end-users feedback were collected and processed in order to identify the most frequent types of use cases, such as in-home health monitoring, house monitoring, fall down detector, automatic door lock, indoor mobility patterns. The architecture we suggest is service oriented, it uses a general representation of data, and protocols for communication and is flexible enough to provide more services than the already existing one. The platform's design for the core subsystem boosts the effectiveness of sensor measurements by applying near real-time processing algorithms via Complex Event Processing engine, or by offering integrated messaging mechanisms. We use the Business Process Management Workflows to define new treatments for the elderly, and to generate medication and medical check alerts. The concept described here opens the way for app developers to extend the platform's functionalities. Developing and deploying the corresponding plug-ins ensure the platform's adaptability requirement.
\end{abstract}

Keywords: Ambient assisted living, service oriented architecture, complex event processing, real-time processing, business workflows, plug-ins

\section{Introduction}

Studies of the future demographic changes, such as Eurostat [13], indicate that the number of senior citizens is increasing all over the world. Under these circumstances, it becomes crucial [15] to find solutions to keep elderly people involved in society, increase their autonomy, and avoid their isolation. In Europe, the Active and Assistive Living (AAL) [1] program was developed in order to improve the life of the elderly. The Assisted living framework's goal is to improve on the existing solutions to elderly care problems as follows:

- Increased mobility will enable the elderly to function autonomously and confidently in a pleasant and secure environment. Self-monitoring of health parameters will lead to improvements in their lifestyle.

\footnotetext{
*Corresponding author. E-mail: ioana.stefan@ siemens.com.
}

- The social networks, dedicated tools and platforms keep the elderly in contact with formal and informal caregivers, family members and friends.

AAL tools have known a major development during last decade. All these concepts address a variety of needs, most of them concentrating on single AAL direction. Rashidi and Mihailidis [32] have overviewed the ways in which AAL concepts were implemented via different technologies and techniques. Another classification of the tools based on AAL requirements has been made in [43]. The surveys conducted so far have allowed for the identification of end user needs across multiple countries.

The AAL Program has encouraged researchers to look for and develop solutions based on Information and Communication Technologies (ICT) oriented toward elderly needs.

AAL systems have to respond, in a personalized and adaptive way, to various users requirements [18]. Since elderly people are less likely to possess the ability to manage and customize complex platforms, the 
AAL compliant platforms must automatically manage the interaction with end users; similarly, all end user graphical interfaces should be as intuitive and suggestive as possible, fulfilling the usability requirements [32] to a large extent.

The proposed concept aims to address multiple requirements such as:

- maintaining health and pleasant living environment for the elderly;

- increase their mobility and safety through fall down detection;

- assist them in their daily activities via reminders;

- connecting the elderly with their caregivers;

- implementing an electronic pill box based on reminders on mobile telephone and sounds;

- enabling them to enjoy an undiminished social life.

The project requirements, surveys and end-users feedback have informed the design of the concept's platform architecture. This has lead to the development of the following use cases: in-home health monitoring, house monitoring, reminders, fall down detector, indoor mobility patterns.

Due to the diversity of the use cases, a natural nonfunctional requirement that our platform offers is good flexibility through the following characteristics:

- incorporation of the Business Process Management (BPM) for processes like new treatments scheduling, electronic pillbox, and medical check reminders;

- multiple configurable dynamic devices for health and home parameters monitoring;

- near real-time processing of data received from devices and generating alerts based on measurements, if needed;

- the possibility to add new custom micro services by allowing the implementation of new custom functionalities to the platform without interrupting running activities.

Ambient Assisted Living Association [27] categorizes stakeholders in three classes: primary, secondary and tertiary users. On the platform we developed, the primary users are the elderly and/or people with medical conditions or disabilities, secondary users are formal caregivers (medical doctors, nurses, home caregivers, external operators) and informal caregivers (family, friends), and tertiary users are application and service providers.
The platform responds to user needs by implementing graphical user interfaces for each type of stakeholder category. All the graphical interfaces are customizable for each single user, in keeping with his/her abilities to interact and manage the system functionalities.

Depending on their business model, the medical or home automation service providers are able to customize our platform through customizable configurations, extensible plug-ins, web services and business process modeler modules in order to reach the specific goals for different variants of service layer agreements.

\section{Related work}

\subsection{AAL platforms use cases}

Different AAL projects [2] stemming out of use cases can be grouped into the following categories:

- Projects that are focused on facilitating communication between the elderly and their informal caregiver without calling each other, including paying virtual visits to the elderly's house.

- Projects emerging from the requirement to monitor health parameters detect and generate alerts based on special situations.

- Projects that implement the environment monitoring and control sensors and actuators to ensure elderly safety and life quality.

- Projects created to improve elderly mobility in and outside their homes.

- Projects oriented towards personal communication (socialization).

- Projects that implement electronic pill box using mobile devices and/or reminders.

A large body of AAL middleware was proposed in various research projects and different papers have classified them [41].

Table 1 is a comparative, category-based list of platforms resulting from different use cases [5,31].

The main goal of the platform proposed in this paper is to deliver multiple services from various use cases, making it expandable i.e. new services can be added, according to the client's needs, and modular i.e., clients can opt for just the services they need.

The platform can be available in different configurations of services, each service having an established price based on the promoted business model [36]. 
Table 1

Use cases based project category

\begin{tabular}{|c|c|c|c|c|c|}
\hline Project & $\begin{array}{l}\text { Facilitating the } \\
\text { elderly and their } \\
\text { informal caregiver } \\
\text { communication }\end{array}$ & $\begin{array}{l}\text { Monitor health } \\
\text { parameters }\end{array}$ & $\begin{array}{l}\text { Environment monitoring } \\
\text { and control sensors and } \\
\text { actuators }\end{array}$ & $\begin{array}{l}\text { Improve elderly mobility } \\
\text { (e.g. falling down detector, } \\
\text { indoor mobility pattern) }\end{array}$ & $\begin{array}{c}\text { Implement electronic } \\
\text { pill box using mobile } \\
\text { devices and/or } \\
\text { reminders }\end{array}$ \\
\hline RelaxedCare [35] & $\mathrm{x}$ & & & & \\
\hline VictoryHome [42] & $\mathrm{x}$ & & & & \\
\hline CARE [9] & & $\mathrm{x}$ & & & \\
\hline HEALTH@HOME [11] & $\mathrm{x}$ & $\mathrm{x}$ & & & \\
\hline REMOTE [8] & $\mathrm{x}$ & $\mathrm{x}$ & & & \\
\hline CLOCKWORK [2] & $\mathrm{x}$ & & $\mathrm{x}$ & & \\
\hline GUIDINGLIGHT [26] & & & & $\mathrm{x}$ & \\
\hline MOBILE.OLD [40] & & & & $\mathrm{x}$ & \\
\hline WALKER [19] & & & & $\mathrm{x}$ & \\
\hline HearMeFeelMe [23] & & & & $\mathrm{x}$ & \\
\hline WeCare [4] & & & & $\mathrm{x}$ & $\mathrm{x}$ \\
\hline PERSONA [41] & $\mathrm{x}$ & & $\mathrm{x}$ & & \\
\hline ReAAL [34] & $\mathrm{x}$ & $\mathrm{x}$ & $\mathrm{x}$ & & \\
\hline Homer [24] & & & $\mathrm{x}$ & & \\
\hline
\end{tabular}

\subsection{Technology}

Grguric [17] has highlighted the main research challenges in developing an AAL solution and platform. The challenge mentioned first is the decomposition of the system in functionality dedicated components (system heterogeneity). This challenge has lately been overcome using Service Oriented Architecture (SOA) [22]. SOA splits business applications into services which represent single processes or functions. On the one hand, services can be part of new applications, which keeps duplication in the enterprise to a minimum. On the other hand, by using SOA approach, the system's reusability is growing because new applications can be easily created. In this way, the enterprise responds to different business requirements.

In SOA a service mostly represents a business task. Services are defined and used based on an interface which hides their implementation [21]. Their interface has the role to integrate the service in the whole business process.

Interfaces are usually stateless and exchange documents. Because the services are easy to update, reconfigure or replace, the system flexibility and adaptability is increased Services can be integrated with other services, making the business logic more complex.

The enterprise architecture based on SOA reduces the development costs, services being reusable and the application programming is done at a high level of abstraction [21]. Furthermore, the manageability and maintainability are better in such systems.

An AAL solution based on SOA architecture is presented in [14] where it is used to create a unified context generation. With AAL systems based on sensors networks, it becomes crucial to maintain the connection between the sensor and the monitoring systems. A way to achieve this and the importance of web-based information and communication technologies is presented in [3]. In the proposed platform, technologies like web services (REpresentational State Transfer REST), and Open Services Gateway initiative (OSGi) are integrated in order to create an extendable and flexible platform.

AAL solutions are aimed at processing the data sent by sensors installed in the elderly's environment or worn by them and at promptly responding to actual conditions, in order to create a safe and comfortable environment for the users. In the paper [10] is presented a way to monitor human behavior based on complex event processing (CEP) solution. The advantage of CEP technique is the power to process a high volume of events in near real-time [25]. Based on row events received from the elderly's environment, the CEP engine detects and takes proper decisions in order to increase the elderly's safety.

In order to plan and manage elderly activities, reminders are created based on generated events. To help monitor their activities, further actions are triggered by the platform's services. Patterns like publish subscriber 
were used to manage and use synchronous and asynchronous communication channels. [29] uses BPEL (Business Process Execution Language) based workflows to define and monitor context behavior. The concept described in this paper defines workflows based on jBPM (JBoss Business Process Management) in order to plan and monitor the elderly (e.g. daily activities, taking medicine plan).

On the market, important mobile devices providers (e.g., Samsung) have created special applications for users' health monitoring [38]. They have developed mobile phones software and hardware to provide users functionalities to measure their heart beat, blood pressure, etc. The option to connect other devices through Bluetooth in order to measure parameters (e.g., body weight) is also available. All these values are saved and accessed by the stakeholders. Furthermore there are web applications to schedule and monitor users activities. Some solutions are cloud oriented, e.g., using Google cloud [16]. The design concept provides also mechanisms for monitoring health parameters, web application being accessed via mobile devices but also from personal computers. Besides health monitoring and users' activity monitoring, the platform proposed in this paper also integrates and provides other type of services, such as domestic monitoring services, fall down service. Moreover, a cloud solution based on OpenShift JBoss cloud services [30] was designed. Depending on the privacy level of the data managed by the service provider managing the proposed platform, it can be configured as Platform as a service (PaaS), Software as a Service, or Infrastructure as a Service variant. Other types of cloud services may be considered, since the architecture is service oriented.

\section{Main results}

The extendable platform concept targets the AAL requirements. These were validated based on end-users surveys and feedback. Their main purposes are to maintain health and pleasant living environment, increase elderly mobility and safety by fall down detection, keep elderly activity plan using reminders, connecting caregivers and elderly, ensure elderly social life. The integrated requirements are covered by the following use cases:

(a) in-house health monitoring (measures elderly health parameters following a regular program established based on their health status, panic button); (b) house monitoring (check home environment status (domotic parameters, water, fire, gas), monitor and control from the platform's user interface doors and windows lock status);

(c) reminders (help the user to remember a scheduled task and supervision of caregivers);

(d) fall down detector (alert a caregiver in case of primary user's falling down);

(e) indoor mobility patterns (identify anomalous behavior and generate alerts)

It should be noted that all the interaction of stakeholders with the platform is realized using a dedicated and customizable user graphic (web and mobile) interface.

The next sections describe the extendable platform architecture in relation to the use cases and the technologies used. The discussion below also refers to the ways in which the platform architecture responds to end-users requirements.

\subsection{Platform outcomes}

Considering that home is where most people receive care, the current functionalities of the platform focus on improving health and home environment. From this perspective, the current platform fits fully meets the stakeholder's needs and expectations.

The survey carried out has provided us with data which has made it possible to prioritize functionalities and focus on more acceptable use cases. Furthermore, since the interest in new devices depends on their technical capabilities and ease of use, the results yielded by the survey results show the most needed functionalities $[33,43]$.

For the carers the platform is customized as a virtual assistant concerned with the health-related matters of caretakers. This flexibility relates to the following respondents measures:

- $69 \%$ of the respondents expressed strong interest in fall detection sensors and other type of medical and automation sensors;

- 78\% use easy mobile phones;

- $91 \%$ of respondents would accept other kind of sensors; all expressed concern regarding possible home intrusion, which made an automatic alarm system highly desirable;

- $69 \%$ were agreeable to the idea of an automatic door lock accessible to caregivers and rescue services; 
- $85 \%$ of the carers claimed that the elderly are deeply affected by social isolation (this has to be corroborated by further data).

The conjoint analysis has proved a high acceptance of a portable sensor $(72 \%)$ from the elderly people and caregivers, who found that wearing a small box which enabled them to get quick help was a good idea [43].

Besides the survey derived requirements stakeholders expect help with reading books, reminders and health related issues.

\subsection{Platform architecture}

The proposed extendable platform was designed to contain the following subsystems (Fig. 1):

- Local Subsystem (LS) that comprises devices installed in elderly home and communicating with the Core Subsystem (CS) via single local fixed (LAN) or wireless (WLAN, cellular) link. LS controls devices and sensors acquire measurement results and transfer them to the CS. It is a combination of hardware and software gateway.

- Nomadic Subsystem (NS) the components of the NS are worn by the caretaker. It enables also out-

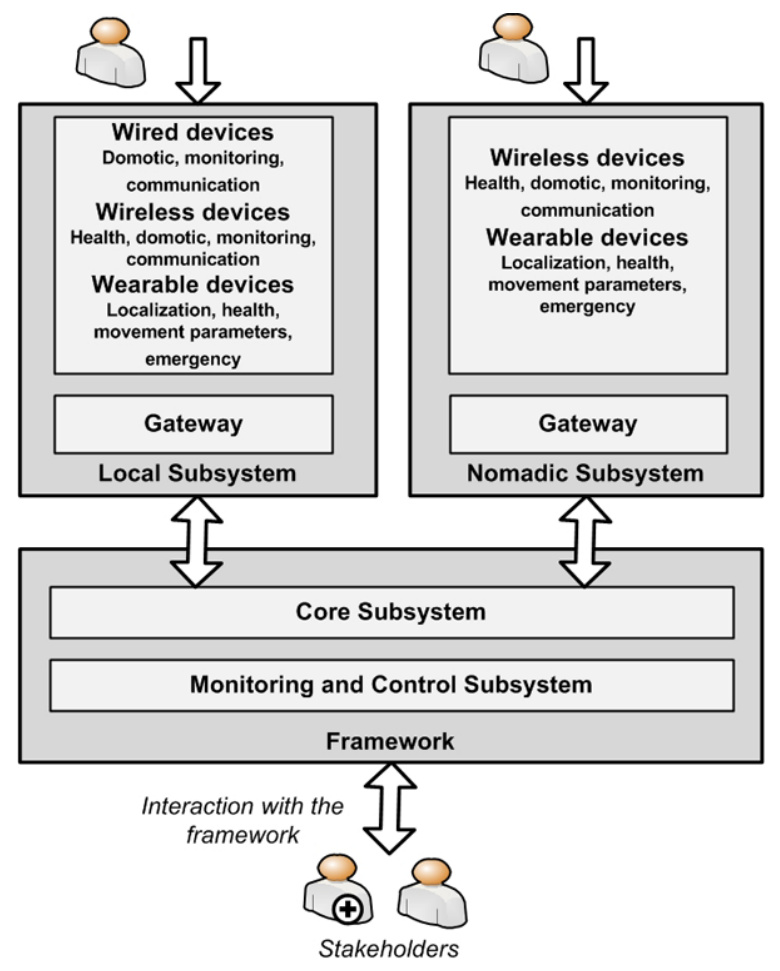

Fig. 1. Platform architecture. door operation in places not covered by the LS. It is based on the mobile devices that are connected using client software to the core subsystem.

- Core Subsystem (CS) is a software component that carries out system control and database functions. It can be installed on a single server machine or into the cloud.

- Monitoring and Control Subsystem (MCS) consists of equipment and software components used by caregivers and system management staff. Its functions include: service control, service data management, presentation of data to end users.

CS and MCS are referred to as the framework. Its goal on the platform's architecture is to implement and control its services, to acquire data sent by LS and NS subsystem, process and save it, and to provide the interaction with the stakeholders involved.

LS and NS subsystems are represented by the wired, wireless and wearable devices and gateways. In order to monitor health and domotic parameters, hardware devices are installed in the user's house. They are connected to a gateway that communicates with the framework in order to allow monitoring and control of all devices from the installed location. In this way, the physiological measurements are combined with context-awareness information, going beyond simple data acquisition.

Gateways send sensor measurements using communication channels like wireless transmission or mobile network to the framework. The goals of the LS gateway and the NS gateway is to gather data from sensors installed in the elderly's home and, respectively, to acquire data from devices that require an increased degree of mobility (e.g., used outside homes).

The designed architecture supports the centralization of the services on a single server or the splitting of major components on different servers (business logic can run on a machine, the database on another, the web services for acquiring data on another, etc.). Each of the architectural components can be offered also by using clouds as PaaS since the implied components are communicating using a message bus that is configured over IP networks. Data originating from the elderly and local devices will be relayed to the Core Subsystem through the gateways. The acquired data is processed by Using default and custom services at Core Subsystem level. 


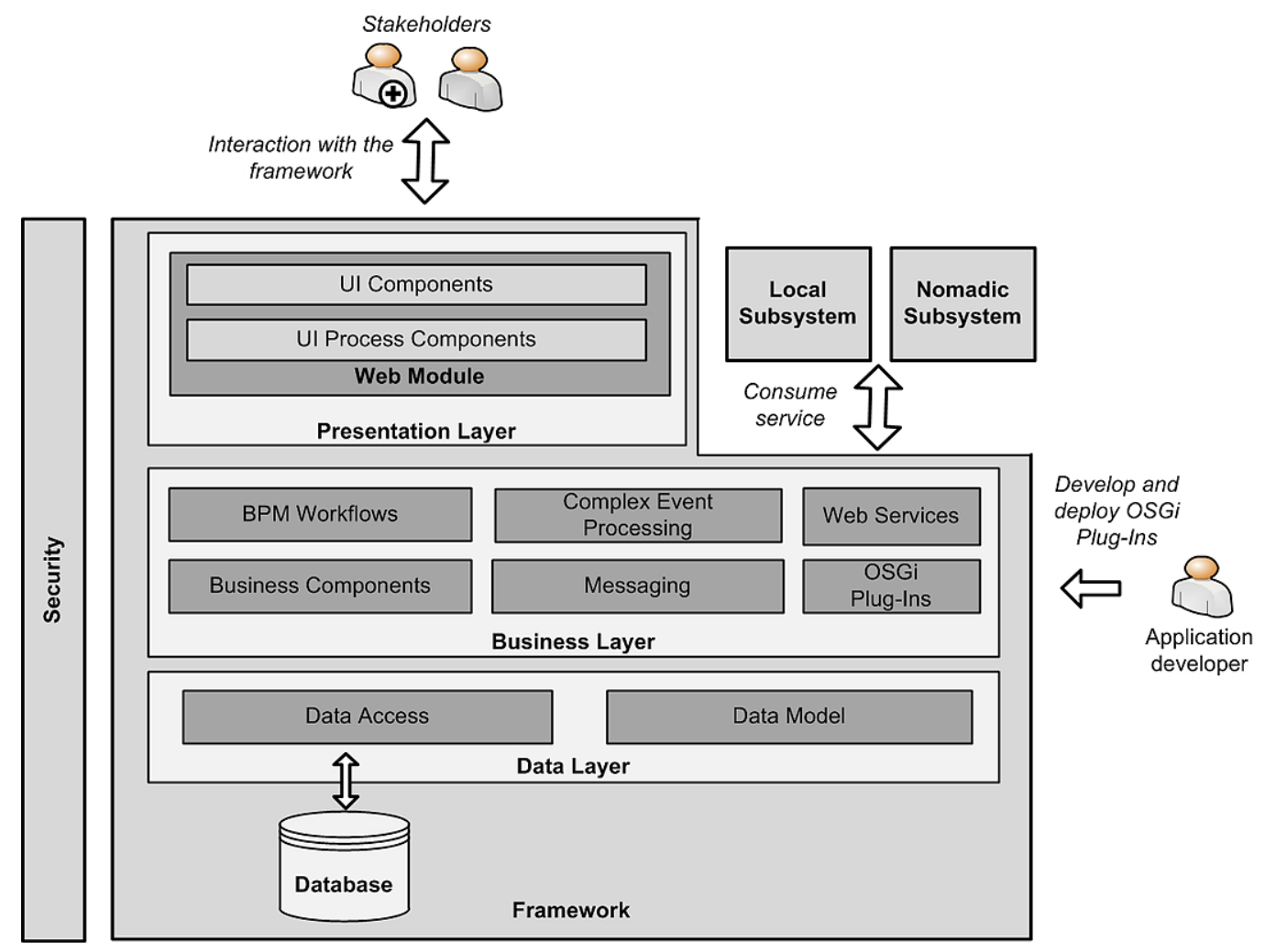

Fig. 2. Framework architecture.

\subsection{Framework architecture}

Into the proposed concept of platform architecture the following logical layers were identified (Fig. 2):

- The Data Layer that is responsible for creating the connection with the database, creating the database model, managing the interaction with the database model.

- The Business Layer responsible for framework functioning logic. On this layer the web services, the business components used to work with data from sensor or database are implemented, processing data from LS and NS subsystems, the business workflows [37], the way messages are transmitted from one module to another, and custom OSGi services.

- The Presentation Layer represents the layer used to define stakeholders' interaction with the framework. It contains a web module which implements the web interface with its processing logic.

Further major components of the architecture are described below.

\subsubsection{Web services}

In order to integrate the LS and NS subsystems with the framework, RESTful web services were used. The proposed platform defines and implements a common Application Programming Interface (API). The programming interface allows sending the data from the devices (e.g., switches, sensors, etc.) to the framework. The gateway reads the raw data, converts it to the defined telegram format based on the custom defined communication protocol, and sends it to the server.

The communication protocol is specified and implemented in order to support the communication between the platform's subsystems. The communication protocol defines structures for different telegram types used to transport the data. Behind main telegram types there are RESTful web services that decode the telegrams, save the measurements, or put them on the common bus which automatically triggers the creation of the measurement events used by Complex Event Processing component.

\subsubsection{Complex event processing}

Complex Event Processing modules were introduced on business level for processing, in near real 


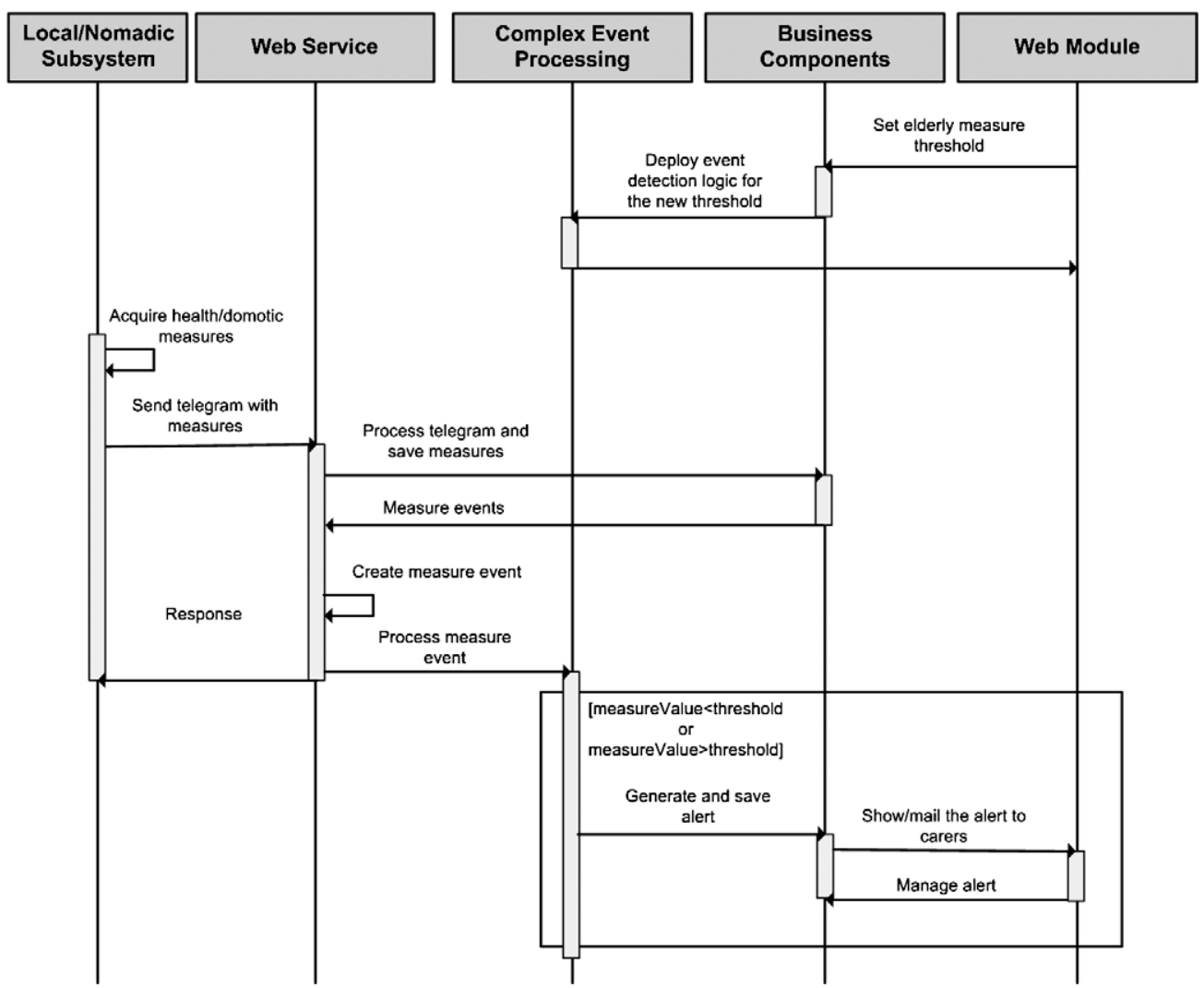

Fig. 3. Abnormal measures values detection.

time, the data sent by LS and NS subsystem. This component was designed for the purpose of optimizing the response time and hence minimize the time necessary to generate alerts and take the necessary actions based on the received sensor measurements (Fig. 3). The presented concept has been validated by using the Esper CEP engine [12].

Event processing logic is designed in an adaptive way which allows the system detect special situations related to the user's health and environment. For example, the stakeholders define from the web interface new elderly measurement thresholds. By using the customized thresholds, positive alerts are raised to other modules.

Based on reference values, actions are triggered automatically by the Business Components using the processing logic of the business layer (Fig. 3).

Using web services, after receiving and saving the sensors measures, different types of events are created in order to be processed by CEP engine.

Figure 3 shows the case of detecting anomalous measurement values (health or home monitoring). The threshold events have a standard format. These are generated when the measured value is outside the normal interval. If this condition is met, then the Business Component generates a new alert for the elderly or caregiver who measured the values. Moreover, the web services are used to record new devices or to update the information about the devices.

Using secure communication, the LS and NS are able to send updates regarding the sensors and devices topology and information about them in such matter that the core automatically learns the changes in topology. Devices can be easily replaced.

\subsubsection{BPM workflows}

Business Workflows are used on the platform to define custom medical workflows (e.g., treatments). Medical caregivers can add new treatments for their elderly care recipients from the web interface (Fig. 4). They can define what medicine should be taken, at what hours, and add a new medical check, if needed, after all the medication is finished.

The Business Workflows are managed, and their status can be checked on the web interface. 


\subsubsection{OSGi plug-ins}

The platform's services based on OSGi dynamic component system allow the use of proprietary functionalities, implemented together with the existing ones without extra downtime for the platform. Developers extend the platform functionalities creating and deploying plug-ins (Fig. 5). From the web interface, stakeholders associate available plug-ins with the end users, based on their specific needs.

After associating the service with the elderly person, the plug-in becomes functional. In order to make it

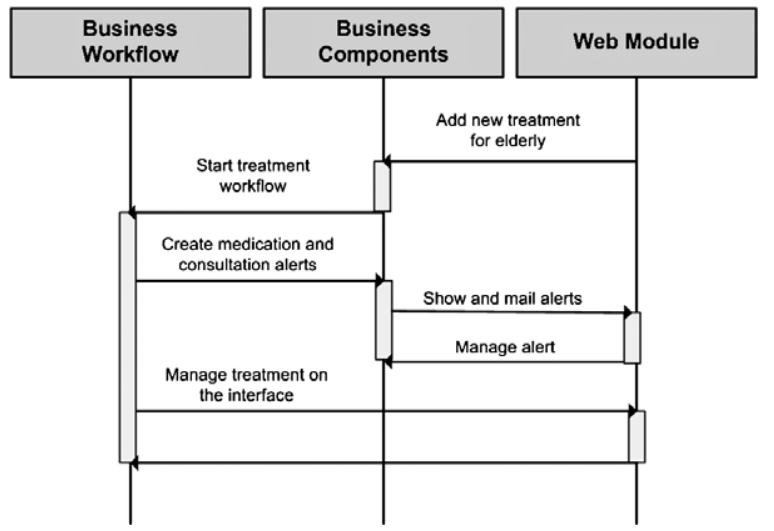

Fig. 4. BPM Workflows. possible to integrate the implemented services at Business Components services level, Web Services were used (REST endpoints). The Application Developer can use all the developed functionalities in order to extend the platform's services without implementing the same functionality twice.

\subsubsection{Messaging}

To exchange events (messages) between Business Layer modules, a communication module that uses the message oriented middleware HornetQ was integrated [20]. This middleware sends telegrams from web services (REST endpoints) to Business Components, transfers row events (created from measures) for Complex Event Processing engine and also detects events (critical situations) to Business Components, in order to generate alerts based on these situations.

The Message Oriented Middleware (MoM) HornetQ has one broker configured at JBoss server level (configuration file). The MoM middleware defines a good communication approach into the distributed systems. Based on this middleware the communication in cloud is simplified.

\subsubsection{Security}

The framework security is a very important issue considered in the design of the proposed extensible platform concept. While the platform is accessed by

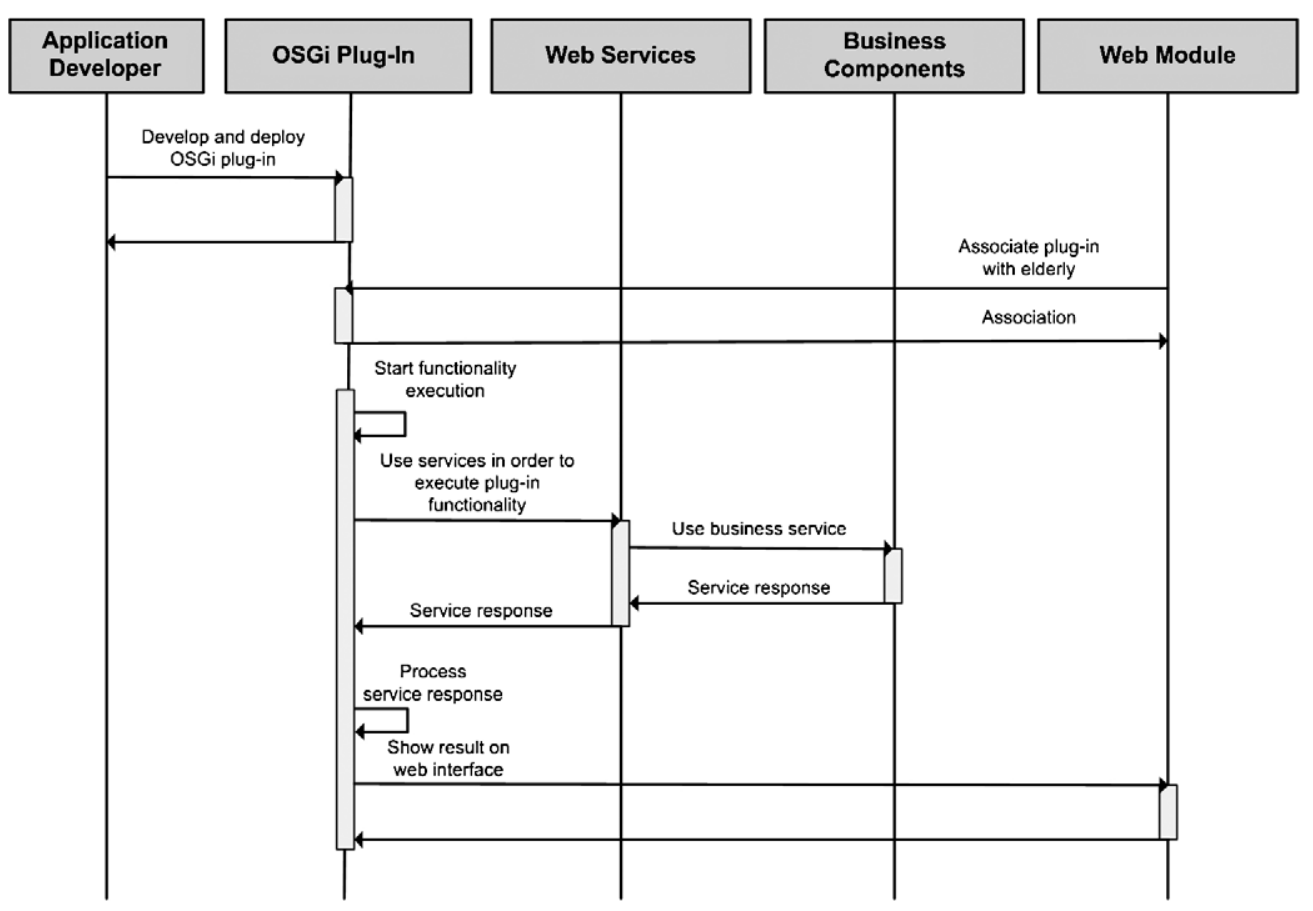

Fig. 5. OSGi Plug-In lifecycle. 
users from a web interface, it is necessary to take into account many of the web platform's security aspects [7]. To ensure authentication, authorization and session management [39] for the framework, different mechanisms (e.g., the application server's security domain) were used.

User authentication and authorization User authentication is done using the username (or the user's email address) and password saved on database after the user registration process. After authentication, based on the associated role, each user is given access to certain services based on the automatic associated permissions. The permissions are customizable at role level and at user level. For beginner users some permission can be deactivated and for the advanced user extra permissions can be activated.

Message hashing and encryption The framework verifies the messages received for validity. Every message received by platform contains a hash field value which is used to authenticate and validate the content of the message. The hash field is compared with values saved at the framework level (database). If this value is saved into the database means that the message is a valid one and it is processed. If the value is not the right one the message is ignored at platform level.

Cache telegrams on data collector devices It is crucial that all data sent by sensors be acquired by the framework. The solution was to implement the cache functionality at the gateways level. When the server is not reachable for a period of time all data sent from sensors to gateway are stored into local caches. After the server becomes available, all the telegrams from the caches are sent from gateways to the framework.

Authenticated dynamic components (plug-ins and web services) All the requests for the web services deployed on the server and plug-ins implemented at the framework level are authenticated. In the platform this problem is taken into account and, as a solution, a field named hash was added to the services requests. This field is used to validate the request and is saved at database level. If the request does not contain a correct hash the request is dropped. Multiple custom hashes can be used.

\subsection{Platform implemented services}

The framework purpose is to provide advanced services to increase elderly mobility in a safe way taking into account the indoor and outdoor environment, to support the elderly people in their daily activities, to create a health and home condition monitoring system, and to maintain their connection with caregivers and family. The provided services can be extended based on the elderly's requirements, adding new customized services through configurations or extendable plug-ins, with minimum effort and without having to restart the system.

The implemented services are integrated also on the web interface.

The web interface was created considering usability rules to respond to all kind of stakeholder requirements, based on their ability to use graphical user interfaces. The elderly's interfaces are created with a simple design in order to increase the usability of the platform.

The next sections describe the main services provided by the framework together with the way they are responding to the user requirements.

\subsubsection{Health monitoring}

Health monitoring use case aims to create the possibility to monitor the elderly people health parameters and to generate alerts when one of these values is outside the normal values.

Based on the generated alerts, the medical staff can take immediate actions (communicate with the elderly, create a new treatment for them) and monitor the results of their actions.

To support this use case, the framework provides the following functionalities on the web interface:

(a) Elderly and caregivers management: elderly account management is done by their caregivers or by administrator users. Every elderly person has associated one or more caregivers, these associations being configurable from the web interface. Elderly information is visualized and managed only by administrators and their caregivers.

(b) Elderly medical profile: available on the web interface, it provides the end user's medical history. This profile contains information about past and current treatments.

(c) Health monitoring device management: in order to monitor their own health parameters, the elderly people can use dedicated devices. These devices are inserted (introduce their MAC/IP, name, etc.) and associated to them on the framework and all measures sent by that device are associated with their respective users. 
(d) Measures management: measured health data is transmitted automatically to the server and stored into the database for further operations.

(e) Data visualization: measures are displayed over a chosen time interval and also report creation is available to users (e.g., carers, elderly, etc.).

(f) Health measurement thresholds: for the health parameters, thresholds need to be introduced into the system settings. Based on these thresholds, the system will trigger an alert message when records are outside these values. Threshold data is hard-coded into the system based on the indications of the user manuals of the devices used for measuring the health parameters. Caregivers will be able to also input personalized threshold limits depending on the health condition of the care recipients under their supervision.

(g) Elderly measurements schedule: caregivers can schedule a monitoring health parameters plan. They can insert events into an agenda, on the basis of which the framework will generate reminders for the elderly care recipients.

(h) Elderly treatment management: the caregiver can insert new medicine plans based on the elderly patients' health condition, plans used to generate medicine taking reminders for them.

\subsubsection{Home monitoring}

In order to increase elderly safety, an intelligent environment was created. The framework checks the home environment status (domestic parameters, water, fire, gas) and generates alerts when one of these values is outside the normal limits. Based on the generated alerts, different types of stakeholders take actions for assuring and improving the elderly's life quality.

To support this use case, the framework provides the following functionalities on the web interface:

(a) Home monitoring device management: devices specialized for home monitoring support the domotic use cases. These devices are installed in elderly environment to monitor principal environment parameters as temperature, water, fire, and gas detection, check and change windows and door lock status.

(b) Measures management: measured health data is transmitted automatically to the server and stored into database for further visualization. Visualization of the data over a chosen time interval and report creation is available to both caregivers and elderly. (c) Devices control: device status can be changed remotely by caregivers and elderly from the web interface.

(d) Home measurement thresholds: works the same as health measurement thresholds presented under health monitoring use case.

(e) Doors and windows status check: the framework generates new alert, if a door or window is open at a given hour every day, to devices associated with the elderly.

\subsubsection{Reminders}

The main goal of reminders use case is to allow users to schedule different activities based on which the platform generates reminders. The reminders are shown on the web interface and also can be sent as emails, according to the user's choice. Also, the reminder functionality is relevant in other use cases like health monitoring (to create measurements schedule, to manage the user's threshold alerts), and to manage all alerts generated by the framework.

To support this use case, the framework provides the following functionalities on the web interface:

(a) Agenda with electronic pillbox: used to create new events based on which the reminders are generated. Caregivers can add events for the elderly people in their care. Users can select which type of alert will trigger the event. Available alert types are: alert message on the dashboard, email, or a combination of both.

(b) Reminders creation: reminders are automatically generated based on event information on the agenda.

(c) Reminders and alerts management: users can confirm, postpone or delete a generated reminder or alert from the web interface (displayed on Dashboard and are colored according to their statuses).

(d) Reminders visualization: carers can visualize elderly reminders and manage them.

(e) Postpone and forward reminders: if the elderly person does not confirm or postpone his/her reminder, it is forwarded to his/her caregiver or to his/her relatives.

\subsubsection{Fall down detector}

The purpose of the fall down detector is to detect when an elderly person falls down and to announce his/her caregiver of this situation.

Based on data received from elderly wearable devices, the NS gateway can detect an elderly falling 
down. At that moment the gateway sends a telegram to the framework to generate a fall down alert. This alert is sent to the elderly's caregivers.

\subsubsection{Indoor mobility pattern}

Indoor mobility pattern use case involves monitoring elderly activity and detecting the moments when he/she does not follow the movement routines about his/her house, in order to announce the caregiver of this situation.

Based on UWB positioning system [6], the data acquired at NS gateway level can detect the situation when the elderly does perform their normal activities. At that moment the gateway sends a telegram to the framework to generate a mobility pattern alert. This alert is sent to the elderly's caregivers.

\subsubsection{Web interface}

The web interface was created in such way as to respond to all stakeholder requirements involved, based on their ability to use such interfaces.

The primary users web interface is simplified and intuitive, allowing users to visualize and manage their alerts, visualize health and environmental measures, reports on measures, communicate with their caregivers or other elderly people, and insert new events on the agenda.

The secondary users web interface is more complex, allowing them to view and manage their own and the elderly's alerts, configure elderly devices (integrate into the platform and set their thresholds), to visualize elderly health profile, to monitor and control elderly home devices, create different reports in order to analyze health parameters and based on these to create a new treatment (for each medicine that should be taken alerts can be generated), insert new events on the agenda for caregiver and his associated elderly.

The tertiary users' web interface is the most complex. These users can access all caregiver functionalities, manage all users' accounts, add custom services for each elderly, set alert's parameters, and create newsletters.

The proposed web interface is an adaptive one. Using the settings, tertiary users can customize what functionalities the primary and secondary users can access based on their experience and ability to use the system. By being intuitive, the interface design increases the platform's usability.

\section{Performance and acceptance}

The proof of concept of the project adopted several phases of testing during the development of the platform. After initial testing in the lab the team carried out extensive trials in target users' home environments, with positive feedback emerging the more people used the system. The project has a fully functional, near-tomarket prototype platform ready.

The platform's web interface and the functionalities designed and implemented that are accessible through the interfaces were tested from the point of view of elderly, caregivers and administrators. The feedback was analyzed and used for subsequent improvements.

The services performance was measured. Multiple raw data sources (sensors) were used and platform messages generated so that the services started the processing and the performance and quality of results was monitored (Table 2).

The trials were organized in three phases. The goal of first phase, which took place in Poland, Romania and Slovenia, was to identify and then rectify major problems and bugs that might come up when using the platform in real-life settings.

The second phase of the trials involved users from Poland (14 users), Romania (16 users) and Slovenia (17 users) who used the platform for 1 or 2 weeks, and some even for over a month. On the whole, the users found the platform useful. One of the main recommendations was related to the sound properties (length, volume, and their meaning).

The third phase of the trials was performed in Romania and Slovenia. Major improvements in the latest version are sound reminders and alerts, synchronization of agenda with the Google or Yahoo calendars, improved user friendliness. Also, the interface for health monitoring was significantly improved for usability and friendliness.

Table 2

Platform performance

\begin{tabular}{ccccc}
\hline CPU RAM & $\begin{array}{c}\text { Number } \\
\text { telegrams }\end{array}$ & $\begin{array}{c}\text { Number } \\
\text { measures }\end{array}$ & $\begin{array}{c}\text { Number } \\
\text { alerts }\end{array}$ & $\begin{array}{c}\text { Time to } \\
\text { process [min] }\end{array}$ \\
\hline 8 & 500 & 2500 & 0 & 2 \\
8 & 1000 & 5000 & 0 & 6 \\
8 & 2000 & 10000 & 0 & 11 \\
8 & 2000 & 20000 & 0 & 15 \\
8 & 500 & 2500 & 2500 & 15 \\
8 & 1000 & 5000 & 5000 & 40 \\
16 & 50000 & 50000 & 50000 & 360 \\
\hline
\end{tabular}




\section{Conclusion}

The purpose of the platform is to address the main AAL requirements by responding to the elderly people's needs. The implemented requirements are covered by the following use-cases: in-home health monitoring, house monitoring, reminders, fall down detector, and indoor mobility patterns. These use cases were identified by means of end-users surveys and feedback.

The platform architecture covers a wide range of hardware devices which collect the data and send it through a gateway to the Core Subsystem. In order to integrate the Local Subsystem and Nomadic Subsystem with the framework, RESTful web services were used. Due to the fact that the designed architecture is service oriented, it uses general representation of data, and protocols for communication that can be extended and used for a larger number of services than the ones analyzed. The subsystems (LS and NS) can also run independently without being connected to the framework.

The enhanced value of the measurements is provided via near real-time processing algorithms using Complex Event Processing engine, or via integrated messaging mechanisms. In this way, the platform's users can be notified regarding the detection of special situations.

The platform functionality is increased by using jBPM Business Workflows technology. These workflows are used with the proposed concept to define new treatments for the elderly, based on which medication and medical check alerts are generated.

Application developers can extend the presented platform functionalities developing and deploying plug-ins, based on the platform's adaptability. OSGi bundles are being deployed without the need of framework restart. The web interface provides a flexible way to customize functionalities by permitting caregivers to associate OSGi plug-ins with the elderly people in their care, according to their needs.

The access to the platform is granted depending on the user roles. Only authorized users have access to restricted areas. After authentication, Each user has access to allowed services based on the automatically associated permissions. The permissions are customizable at role level and, also at user level. In order to increase the web interface's usability and to ensure framework security, some permission can be deactivated for novice users, while additional permissions can be activated for the advanced users..
The modern methods and technologies (complex event processing, business process modeling, web services, etc.) were used to design AAL requirements (easily manage and access the elderly related data) into a project concept. The concept developed has been tested successfully by implementing the platform with a large number of functionalities [28].

\section{Acknowledgements}

This paper is supported by the NITICS project, Networked InfrasTructure for Innovative home Care Solutions (http://nitics.eclexys.com/). NITICS is a Small or medium-scale focused research project (STREP) funded within the European Grant AAL Joint Programme, contract number: AAL-20.

\section{References}

[1] Active and assisted living programme, http://www.aal-europe. eu/, accessed January 2017.

[2] Active and assisted living programme projects, http://www. aal-europe.eu/our-projects/, accessed January 2017.

[3] N. Agoulmine, P. Ray and T.H. Wu, Efficient and cost-effective communications in ubiquitous healthcare: Wireless sensors, devices and solutions, IEEE Communications Magazine 50 (2012), 90-91. doi:10.1109/MCOM.2012.6194387.

[4] H.O. Alemdar and C. Ersoy, WeCare: Wireless enhanced healthcare, in: Mediterranean Conference on Medical and Biological Engineering and Computing, IFMBE Proceedings, Vol. 29, 2010, pp. 855-858.

[5] J.A. Álvarez-García, P. Barsocchi, S. Chessa and D. Salvi, Evaluation of localization and activity recognition systems for ambient assisted living: The experience of the 2012 EvAAL competition, Journal of Ambient Intelligence and Smart Environments 5 (2013), 119-132.

[6] A. Badawika and J. Kolakowski, UWB positioning system architecture based on paired anchor nodes, in: 20th International Conference on Microwaves, Radar, and Wireless Communications, Gdansk, 2014, pp. 1-4.

[7] L. Bass, P. Clements and R. Kazman, Software Architecture in Practice, 3rd edn, SEI Series in Software Engineering, Addison Wesley Professional, 2012.

[8] A. Bekiaris, A. Mourouzis and N. Maglaveras, The REMOTE AAL project: Remote health and social care for independent living of isolated elderly with chronic conditions, in: Universal Access in Human-Computer Interaction. Context Diversity, Lecture Notes in Computer Science, Vol. 6767, 2011, pp. 131140. doi:10.1007/978-3-642-21666-4_15.

[9] A.N. Belbachir, T. Lunden, P. Hanak, F. Markus, M. Bottcher and T. Mannersola, Biologically-inspired stereo vision for elderly safety at home, Elektrotechnik und Informationstechnik 127 (2010), 216-222. doi:10.1007/s502-010-0750-1. 
[10] R. Culmone, M. Falcioni, P. Giuliodori, E. Merelli, A. Orru and M. Quadrini, AAL domain ontology for event-based human activity recognition, in: 2014 IEEE/ASME 10th International Conference on Mechatronic and Embedded Systems and Applications (MESA), Senigallia, 2014, pp. 1-6.

[11] M. Donati, T. Bacchillone, L. Fanucci, S. Saponara and F. Costalli, Operating protocol and networking issues of a telemedicine platform integrating from wireless home sensors to the hospital information system, Journal of Computer Networks and Communications 2013 (2013), 781620.

[12] EsperTech Event Series Intelligence, http://www.espertech. com/products/esper.php, accessed January 2016.

[13] Eurostat, International Day of Older Persons - 1 Out of Every 8 Persons in the EU Could Be 80 or Above by 2080 - Elderly People Less at Risk of Poverty or Social Exclusion, European Commission, Brussels, 2015.

[14] A. Forkan, I. Khalil and Z. Tari, CoCaMAAL: A cloudoriented context-aware middleware in ambient assisted living, Future Generation Computer Systems 35 (2014), 114-127. doi:10.1016/j.future.2013.07.009.

[15] FuturAge: A road map for ageing research, http://futurage. group.shef.ac.uk/home.html, accessed January 2017.

[16] Google Could, https://cloud.google.com/, accessed January 2017.

[17] A. Grguric, ICT towards elderly independent living, http:// www.enhemsbuildings.fer.hr/_download/repository/A._ Grguric,_rad_KDI.pdf, accessed January 2016.

[18] A. Grguric, D. Huljenic and M. Mosmondor, 2015 IEEE International Conference on AAL Ontology: From Design to Validation, Communication Workshop (ICCW), London, 2015, pp. 234-239.

[19] Happy Walker, http://www.happyassistedliving.com/, accessed January 2017.

[20] HornetQ, http://hornetq.jboss.org/, accessed January 2016.

[21] F. Jammes and H. Smit, Service-oriented paradigms in industrial automation, IEEE Transactions on Industrial Informatics 1 (2005), 62-70. doi:10.1109/TII.2005.844419.

[22] N. Josuttis, SOA in Practice: The Art of Distributed System Design, O’Reilly Media, 2007.

[23] A. Konttila, M. Harjumaa, S. Muuraiskangas, M. Jokela and M. Isomursu, Touch n' tag: Digital annotation of physical objects with voice tagging, Journal of Assistive Technologies 6 (2012), 24-37. doi:10.1108/17549451211214337.

[24] J. Kropf, L. Roedl and A. Hochgatterer, A modular and flexible system for activity recognition and smart home control based on nonobtrusive sensors, in: 6th International Conference on Pervasive Computing Technologies for Healthcare (PervasiveHealth) and Workshops, San Diego, CA, 2012, pp. 245-251.

[25] D.C. Luckham, Rapide: A language and toolset for simulation of distributed systems by partial orderings of events, 1996.

[26] C. Nedopil, C. Schauber and S. Glende, Creating user-friendly healing environments with adaptable lighting for senior citizens, in: Engineering Psychology and Cognitive Ergonomics. Applications and Services, Lecture Notes in Computer Science, Vol. 8020, 2013, pp. 365-373. doi:10.1007/978-3-64239354-9 40.
[27] C. Nedopil, C. Schauber and S. Glende, Knowledge Base AAL Stakeholders and Their Requirements, Ambient Assisted Living Association, Brussels, 2013.

[28] Networked InfrasTructure for Innovative home Care Solutions project, http://nitics.eclexys.com/, accessed January 2017.

[29] OpenAAL - The open source middleware for ambient-assisted living, http://openaal.org/, accessed January 2017.

[30] OpenShift Cloud, https://www.openshift.com, accessed January 2017.

[31] R. Phull, R. Liscano and A. Mihailidis, Comparative analysis of prominent middleware platforms in the domain of ambient assisted living (AAL) for an older adults with dementia (OAwD) scenario, Procedia Computer Science 83 (2016), 537-544. doi:10.1016/j.procs.2016.04.252.

[32] A. Queiros, A. Silva, J. Alvarelhao, N.P. Rocha and A. Teixeira, Usability, accessibility and ambient-assisted living: A systematic literature review, Universal Access in the Information Society 14 (2015), 57-66. doi:10.1007/s10209-013-0328-x.

[33] P. Rashidi and A. Mihailidis, A survey on ambient-assisted living tools for older adults, IEEE Journal of Biomedical and Health Informatics 17 (2013), 579-590. doi:10.1109/JBHI. 2012.2234129.

[34] ReAAL, http://www.cip-reaal.eu/home/, accessed January 2017.

[35] RelaxedCare project, http://www.relaxedcare.eu/de/, accessed January 2016.

[36] D. Rudel, A. Consoli, J. Ayadi, Ł. Malicki, C.L. Aldea, O. Cramariuc, S. Smidtas and T. Didi, D6.5: Demonstrator sessions, 2014, http://deliverables.aal-europe.eu/call-5/nitics.

[37] L. Rusu, B. Cramariuc, D. Benta and M. Mailat, Implementing BPMN 2.0 scenarios for AAL@ Home solution, International Journal of Computers Communications \& Control 10 (2015), 230-237. doi:10.15837/ijccc.2015.2.1754.

[38] SHealth application, Samsung, http://shealth.samsung.com/, accessed January 2017.

[39] S. Subashini and V. Kavitha, A survey on security issues in service delivery models of cloud computing, Journal of Network and Computer Applications 34 (2011), 1-11. doi:10.1016/j. jnca.2010.07.006.

[40] B. Tarnauca, D. Puiu, S. Nechifor and V. Comnac, Using complex event processing for implementing a geofencing service, in: 2013 IEEE 11th International Symposium on Intelligent Systems and Informatics (SISY), Subotica, 2013, pp. 391-396. doi:10.1109/SISY.2013.6662608.

[41] M.R. Tazari, F. Furfari, J.-P.L. Ramos and E. Ferro, The PERSONA service platform for AAL spaces, in: Handbook of Ambient Intelligence and Smart Environments, Springer, 2010, pp. 1171-1199. doi:10.1007/978-0-387-93808-0_43.

[42] VictoryaHome project, http://www.victoryahome.eu/be-wellvictoryahome-create-possibilities.5480465.html, accessed January 2017.

[43] I. Zielinska, L. Malicki, N.S. Brencic, A. Consoli, J. Ayadi, L. Gilardi, O. Cramariuc, D. Stanciu, T. Didi and S. Smidtas, D2.1: The results of the multi-national survey, 2013, http:// deliverables.aal-europe.eu/call-5/nitics. 